\title{
Can toxicities induced by antituberculosis drugs be better managed in diabetic patients?
}

\author{
To the Editor:
}

The converging epidemics of tuberculosis and diabetes mellitus worldwide have immense healthcare implications [1]. Diabetes mellitus adversely impacts tuberculosis treatment outcomes, largely as delayed bacteriological conversion and lower cure, as well as higher relapse and mortality. Worsened drug resistance scenarios may also result. In addition, diabetic patients experience a higher risk of toxicities induced by drugs used for treating drug-susceptible tuberculosis and multidrug-resistant tuberculosis (table 1) [1,2]. Clearly, these adverse reactions merit better management.

Diabetes mellitus is a metabolic disease with a strong pathogenetic background of chronic inflammation. It is now recognised that hyperglycaemia and advanced glycation end-products, resulting from poor control of the metabolic derangement inherent to the disease, are conducive to inappropriate oxidative stress and mitochondrial dysfunction. These mechanisms underlie the accelerated or relentless development of diabetic complications, especially those pertaining to the cardiovascular and neurological systems [3]. Recently, fatty change and fibrosis in the diabetic liver, and diabetic nephropathy have also been linked to such pathogenetic basis. Oxidative stress and mitochondrial dysfunction have been shown to be responsible for isoniazid-induced neurotoxicity and hepatotoxicity in the rat model [4]. Similarly, these mechanisms have also been implicated in the pathogenesis of toxicities induced by pyrazinamide, aminoglycosides and fluoroquinolones in different experimental models.

Regarding the treatment of multidrug-resistant tuberculosis, linezolid use has been shown to incur peripheral neuropathy, even at a low daily dose. Using biomarkers to assess mitochondrial function, in form of translational competence, the serum trough concentration of linezolid was found to correlate positively with mitochondrial dysfunction in patients with extensively drug-resistant tuberculosis [5]. Use of bedaquiline, delamanid, clofazimine or a later-generation fluoroquinolone can be potentially associated with risk of cardiotoxicity. Oxidative stress and mitochondrial dysfunction are the putative mechanisms underlying cardiotoxicity induced by a variety of drugs [6]. Using the zebrafish model, clofazimine-induced cardiotoxicity was shown to be associated with oxidative-stress response in the host ( J.Y. Chan and co-workers, unpublished results presented at the Interscience Conference on Antimicrobial Agents and Chemotherapy, San Diego, CA, USA, 2015).

Is there any upcoming insight that might help to better manage adverse reactions induced by antituberculosis drugs in diabetic patients? Poorly controlled diabetes mellitus increases the risk of active tuberculosis, and also results in the propensity for developing medical comorbidities [3]. Thus optimal management of the underlying metabolic derangement constitutes the cornerstone strategy to reduce the risk of developing diabetic complications that can aggravate the aftermath of antituberculosis drug-induced toxicities, due to the overlapping/shared pathogenetic mechanisms in organ injury. As there is currently a lack of robust clinical data regarding the association between risk of antituberculosis drug-induced toxicities and diabetic control, relevant research is of great importance and sorely needed.

Given the severity of the adverse reactions induced by antituberculosis drugs often encountered in diabetic patients [2], a vigilant, proactive and intensive approach in their management must be adopted. A few examples help to reiterate the message. Diligent monitoring of development of tinnitus (by symptom assessment) and auditory acuity (by audiometry) to detect aminoglycoside-induced toxicity is highly beneficial.

@ERSpublications

Elucidating mechanisms of antituberculosis-drug-induced toxicities in diabetic patients helps in clinical management http://ow.ly/92vA30cAlBW

Cite this article as: Yew WW, Chan DP, Leung CC, et al. Can toxicities induced by antituberculosis drugs be better managed in diabetic patients? Eur Respir J 2017; 50: 1700409 [https://doi.org/10.1183/ 13993003.00409-2017]. 
TABLE 1 Major adverse reactions induced by antituberculosis drugs

\begin{tabular}{llll} 
Adverse reaction & $\begin{array}{c}\text { Rate }(\%) \text { in patients with DM } \\
\text { versus without }\end{array}$ & Incriminated drug(s) & Associated mechanism(s) \\
\hline Nephrotoxicity & 73.7 versus $30.0^{*}$ & $\mathrm{Am}, \mathrm{Km}, \mathrm{Cm}, \mathrm{S}$ & $\mathrm{D}$ \\
Hypothyroidism & 55.6 versus $12.5^{*}$ & $\mathrm{PAS}, \mathrm{Eto} / \mathrm{Pto}$ & $\mathrm{D}$ \\
Ototoxicity & 56.5 versus 32.0 & $\mathrm{Am}, \mathrm{Km}, \mathrm{Cm}, \mathrm{S}$ & $\mathrm{D}$ \\
Restlessness & 36.6 versus $16.8^{*}$ & $\mathrm{Cs}, \mathrm{FQ}, \mathrm{H}$ & $\mathrm{D}$ \\
Peripheral neuropathy & 34.1 versus 21.0 & $\mathrm{Lzd}, \mathrm{H}, \mathrm{Cs}, \mathrm{Eto} / \mathrm{Pto}, \mathrm{Km}, \mathrm{Am}, \mathrm{Cm}, \mathrm{FQ}$ & $\mathrm{D}$ \\
Nausea and vomiting & 26.8 versus 17.7 & $\mathrm{Eto} / \mathrm{Pto}, \mathrm{PAS}, \mathrm{Z}, \mathrm{R}, \mathrm{Clo}, \mathrm{E}$ & $\mathrm{D}$ \\
Skin rash & 24.4 versus 20.1 & $\mathrm{H}, \mathrm{R}, \mathrm{Z}, \mathrm{S}, \mathrm{E}, \mathrm{PAS}, \mathrm{Cs}, \mathrm{Eto} / \mathrm{Pto}$ & $\mathrm{I} / \mathrm{H}$ \\
Liver injury & 17.1 versus 15.0 & $\mathrm{H}, \mathrm{Z}, \mathrm{R}, \mathrm{Eto} / \mathrm{Pto}, \mathrm{PAS}, \mathrm{FQ}$ & $\mathrm{I}$ H; D(?) \\
Psychiatric disorders & 18.0 versus 19.0 & $\mathrm{Cs}, \mathrm{FQ}, \mathrm{H}, \mathrm{Eto} / \mathrm{Pto}$ & $\mathrm{D}$ \\
Joint pain & 12.2 versus 8.9 & $\mathrm{Z}, \mathrm{FQ}, \mathrm{E}, \mathrm{H}$ & $\mathrm{D} ; \mathrm{l} / \mathrm{H}(?)$ \\
\hline
\end{tabular}

DM: diabetes mellitus; Am: amikacin; Cm: capreomycin; Clo: clofazimine; Cs: cycloserine; E: ethambutol; Eto/Pto: ethionamide/prothionamide; FQ: fluoroquinolone; $\mathrm{H}$ : isoniazid; $\mathrm{Km}$ : kanamycin; Lzd: linezolid; PAS: para-aminosalicylic acid; R: rifampicin; S: streptomycin; Z: pyrazinamide; D: dose-dependence; I/H: idiosyncrasy/hypersensitivity. ${ }^{\#}$ : drugs listed in descending order of likelihood; *: p<0.05 [1, 2].

Likewise, inquiry of symptom of paraesthesia followed by nerve conduction testing would enable early detection of peripheral neuropathy. Prudent monitoring for cardiotoxicity (usually by assessment of electrocardiographic QTc interval) during the use of bedaquiline and other agents with potential cardiotoxicity is mandatory. Drug-drug interactive toxicities merit due awareness and remedy. In diabetic subjects with multidrug-resistant tuberculosis receiving metformin and linezolid, lactic acidosis might result from the interactive toxicity of these two drugs. In patients with HIV infection or malaria, concomitant to diabetestuberculosis, clinically relevant interactive toxicities among the therapeutics for these diseases is anticipated.

Assessment of pharmacokinetics can help in the clinical management of adverse drug reactions. Manipulation of the dosing schedule, such as conversion from daily to three-times-weekly administration of linezolid to reduce the serum trough concentration of the drug may help forestalling neurotoxicity [7]. Therapeutic monitoring of serum drug concentrations in selected patients, particularly elderly diabetics with comorbidities, to optimise drug exposure, to manage drug-drug interactions, and to ameliorate drug toxicity [8] would favourably address the efficacy versus toxicity of antituberculosis drugs.

The merits of programmatic management of both tuberculosis and diabetes in close collaboration cannot be overemphasised. In addition, the contributing roles of other specialists should be appreciated. Furthermore, attempt is currently being made to explore the targeted use of antioxidants and beyond in diseases with pathogenetic mechanisms predominantly involving redox imbalance and mitochondrial dysfunction [9]. This new strategy is likely pertinent in managing the toxicities induced by antituberculosis drugs in patients with diabetes mellitus. Recently, modification of the molecular structure of clofazimine was found to minimise its toxicity, alongside preservation of its efficacy [10]. Hopefully these new endeavours, utilising supermolecules or other approaches, may become viable therapeutic options in tuberculosis patients, especially those with concomitant diabetes mellitus, in the future.

Wing Wai Yew ${ }^{1}$, Denise P. Chan ${ }^{1}$, Chi Chiu Leung ${ }^{2}$, Ying Zhang ${ }^{3}$, Ruibing Wang ${ }^{4}$, Phoebe $\mathrm{Ng}^{4}$ and Simon M. Lee ${ }^{1}$ Stanley Ho Centre for Emerging Infectious Diseases, Faculty of Medicine, The Chinese University of Hong Kong, Hong Kong, China. ${ }^{2}$ Tuberculosis and Chest Service, Centre for Health Protection, Department of Health, Hong Kong, China. ${ }^{3}$ Department of Molecular Microbiology and Immunology, Bloomberg School of Public Health, Johns Hopkins University, Baltimore, MD, USA. ${ }^{4}$ State Key Laboratory of Quality Research in Chinese Medicine and Institute of Chinese Medical Sciences, University of Macau, Macao, China.

Correspondence: Denise P. Chan, Rm 207, 2/F, Stanley Ho Centre for Emerging Infectious Diseases, Postgraduate Education Centre, Prince of Wales Hospital, Shatin, Hong Kong. E-mail: denisechan@cuhk.edu.hk

Received: Feb 252017 | Accepted after revision: May 012017

Conflict of interest: Disclosures can be found alongside this article at erj.ersjournals.com

Acknowledgement: Li Ka Shing Institute of Health Sciences is gratefully acknowledged for providing technical support to this research

\section{References}

1 Siddiqui AN, Khayyam KU, Sharma M. Effect of diabetes mellitus on tuberculosis treatment outcome and adverse reactions in patients receiving directly observed treatment strategy in India: a prospective study. Biomed Res Int 2016; 2016: 7273935. 
2 Munoz-Torrico M, Caminero-Luna J, Migliori GB, et al. Diabetes is associated with severe adverse events in multidrug-resistant tuberculosis. Arch Bronconeumol 2017; 53: 245-250.

3 Fiorentino TV, Prioletta A, Zuo P, et al. Hyperglycemia-induced oxidative stress and its role in diabetes mellitus related cardiovascular diseases. Curr Pharm Des 2013; 19: 5695-5703.

4 Ahadpour M, Eskandari MR, Mashayekhi V, et al. Mitochondrial oxidative stress and dysfunction induced by isoniazid: study on isolated rat liver and brain mitochondria. Drug Chem Toxicol 2016; 39: $224-232$.

5 Song T, Lee M, Jeon HS, et al. Linezolid trough concentrations correlate with mitochondrial toxicity-related adverse events in the treatment of chronic extensively drug-resistant tuberculosis. EBioMedicine 2015; 2: 1627-1633.

6 Varga ZV, Ferdinandy P, Liaudet L, et al. Drug-induced mitochondrial dysfunction and cardiotoxicity. Am J Physiol Heart Circ Physiol 2015; 309: H1453-H1467.

7 Chang KC, Yew WW, Cheung SW, et al. Can intermittent dosing optimize prolonged linezolid treatment of difficult multidrug-resistant tuberculosis. Antimicrob Agents Chemother 2013; 57: 3445-3449.

8 Alsultan A, Peloquin CA. Therapeutic drug monitoring in the treatment of tuberculosis: an update. Drugs 2014; 74: 839-854.

9 Enns GM. Treatment of mitochondrial disorders: antioxidants and beyond. J Child Neurol 2014; 29: 1235-1240.

10 Li S, Chan JY, Li Y, et al. Complexation of clofazimine by macrocyclic cucurbit[7]uril reduced its cardiotoxicity without affecting the antimycobacterial efficacy. Org Biomol Chem 2016; 14: 7563-7569.

Copyright @eERS 2017 